\title{
Minimally Invasive Access to the Posterior Cranial Fossa: An Anatomical Study Comparing a Retrosigmoidal Endoscopic Approach to a Microscopic Approach
}

\author{
Jason Van Rompaey ${ }^{1}$ Carrie Bush ${ }^{1} \quad$ Brian McKinnon ${ }^{1}$ Arturo C. Solares ${ }^{1}$ \\ ${ }^{1}$ Georgia Skull Base Center, Department of Otolaryngology, Georgia \\ Health Sciences University, Augusta, Georgia, United States \\ J Neurol Surg A 2013;74:1-6. \\ Address for correspondence and reprint requests Jason Van Rompaey, \\ Georgia Skull Base Surgery Center, Department of Otolaryngology, \\ Georgia Health Sciences University, 1120 15th Street, Augusta, GA \\ 30912-4060, United States (e-mail: jvanrompaey@georgiahealth.edu).
}

\begin{abstract}
Keywords

- cerebellopontine angle

- keyhole approach

- endoscopy-assisted microsurgery

- endoscopic retrosigmoidal approach

- endoscopy-assisted microsurgery

- intracranial tumor

Objectives The central location and complex neurovascular structures of the posterior cranial fossa make tumor resection in this region challenging. The traditional surgical approach is a suboccipital craniotomy using a microscope for visualization. This approach necessitates a large surgical window and cerebellar retraction, which can result in patient morbidity. With the advances in endoscopic technology, minimally invasive access to the cerebellopontine angle can be achieved with minimal manipulation of uninvolved structures, reducing the complications associated with the suboccipital approach.

Methods An endoscopic and microscopic approach was completed on anatomic specimens. To access the central structures of the posterior cranial fossa, a retrosigmoidal approach was undertaken. A keyhole craniotomy was made in the occipital bone posterior to the junction of the transverse and sigmoid sinuses. The endoscope was advanced and photographs were obtained for review. The exposure was compared with that obtained with a microscope.

Results The endoscopic retrosigmoidal approach to the posterior cranial fossa provided increased exposure to the midline structures while minimizing the surgical window. The relevant anatomy was identified without difficulty.

Conclusion An endoscopic retrosigmoidal approach to the midline structures of the posterior cranial fossa is anatomically feasible. The morbidity associated with retraction of the cerebellum could possibly be avoided, improving patient outcomes. Retrosigmoidal endoscopy provides access to anatomical structures that is not possible using a microscope in a suboccipital approach. Further understanding of the endoscopic anatomy of the posterior fossa can allow for advances in cranial base surgery with improved safety and efficacy.
\end{abstract}

\section{Introduction}

The cerebellopontine (CP) angle is located in the anatomically dense skull base and can be associated with a wide variety of pathology that may require surgical intervention. ${ }^{1-4}$ The pathology may include neurovascular compression and aneurysms. $^{1-3,5}$ However, the most common disease requiring surgical excision are tumors. ${ }^{1,5}$ The limited access to this region has prompted investigators to innovate methods of received

August 29, 2011

accepted

March 2, 2012

published online

January 4, 2013
C 2013 Georg Thieme Verlag KG Stuttgart · New York
DOI http://dx.doi.org/ 10.1055/s-0032-1330119. ISSN 2193-6315. 
creating an adequate surgical corridor while limiting the damage and sacrifice of surrounding structures.

As with any surgical procedure, an adequate window of visualization is key to providing operative success. The most commonly used surgical approach for accessing the $\mathrm{CP}$ angle has been a lateral suboccipital craniotomy, also known as the retrosigmoidal approach. ${ }^{2,4}$ This creates a direct path along the petrous portion of the temporal bone toward the CP angle. By convention, the operative microscope has been used as the primary instrument of visualization to identify the fine anatomical structures, resulting in successful tumor extirpation and neural decompressive treatments.

However, for the microscope to obtain an adequate surgical corridor, retraction of the cerebellum is required ${ }^{4}$ with the consequence of cerebellar manipulation, increasing the possibility of morbidity. ${ }^{5-7}$

This associated morbidity has prompted the investigative use of endoscopy in the application of CP access. ${ }^{5}$ Endoscopy is applied to many surgical disciplines, enabling adequate visualization while minimizing injury to surrounding anatomy. Endoscopic surgery to the skull base may obviate many of the drawbacks with open approaches. ${ }^{8}$ The deep and complex nature of $\mathrm{CP}$ anatomy makes this region an attractive candidate for endoscopy-assisted surgery. ${ }^{4,5}$

The purpose of this study is to evaluate the extent of surgical visualization that can be achieved with an endoscopic retrosigmoidal approach to the $\mathrm{CP}$ angle. A comparison to the views created by a microscope and an outline of the procedures taken to access the various structures will be made.

\section{Materials and Methods}

\section{Specimens}

Two anatomic specimens were obtained to review the relevant anatomy. After being preserved in alcohol, they were injected with colored latex highlighting the arterial and venous vasculature. The procedure for head injection was followed according to the guidelines set by Sanan et al. ${ }^{9}$

\section{Retrosigmoidal Keyhole Craniotomy}

The craniotomy for the retrosigmoidal approach is made with the intention of creating the ideal path to the $\mathrm{CP}$ angle while avoiding hemorrhage of the transverse and sigmoid sinuses. A lateral suboccipital craniotomy allows a direct path to the $\mathrm{CP}$ angle with minimal retraction of the cerebellum. This approach is parallel and inferoposterior to the superior petrosal sinus and the petrous portion of the temporal bone.

To preserve the transverse and sigmoid sinuses, landmarks for the keyhole craniotomy were first established. Raso and Gusmao ${ }^{10}$ provided guidelines for optimal placement of the retrosigmoidal craniotomy. Following these directions, a line was made from the inion to the superior border of the zygomatic arch. This line approximates the inferior border of the transverse sinus along the occipital bone. Another line was then made concurrent with the digastric fossa posterior to the mastoid process. This approximates the border of the sigmoid sinus. The intersection of the two lines was the inferior border of the intersection of the sigmoid and transverse sinus. These lines also were the superior and superolateral borders of the craniotomy. A sagittal incision of sufficient size was made medial to the intersecting lines to allow a $25 \mathrm{~mm}$ craniotomy. Skin retractors and a periosteal elevator were used to reveal the occipital bone. A high-speed drill with a diamond bit was then used to obtain a burr hole. A cutting tool was then taken and the small $25 \mathrm{~mm}$ bone flap was created. The dura was cut along the borders of the craniotomy and reflected superiorly.

\section{Dissection Technique}

A zero-degree rod endonasal endoscope (Karl Storz, Tuttlingen, Germany) was inserted into the craniotomy. The endoscope was placed on a stand, permitting bimanual microdissection technique of the contents of the posterior fossa. Arachnoid mater was removed surrounding the contralateral cranial nerve $(\mathrm{CN}) \mathrm{XI}$ and posterior brain stem. The endoscope was then directed to the ipsilateral side and CN VII, VIII, IX, X, and XI were visualized after removal of the obscuring arachnoid mater. The endoscope was placed superiorly in relation to the craniotomy. This enabled access to CN V and VI without damaging cranial nerves VII and VIII. CN IV was then dissected at the tentorial incisure. This nerve was dissected medial until the origin was reached, inferior to the inferior colliculus.

Anterior to $\mathrm{CN} \mathrm{V}$, the petrosal vein was then divided from the tentorium, allowing the endoscope unobstructed penetration deeper into the posterior fossa toward the clivus. This permitted better visualization of CN III, which was then dissected. Advancing the endoscope along the petroclival border into the sella allowed direct visualization of the pituitary stalk and mamillary bodies. The arachnoid mater was dissected from these structures to allow an unobstructed view via a small surgical window. Digital high-definition photographs were then obtained of all the relevant structures.

An operating microscope (Carl Zeiss Meditec, Inc. Dublin, California) was then used to view the same anatomy through the same $25 \mathrm{~mm}$ craniotomy used for endoscopy. Photographs were also obtained for a comparison to the endoscopic approach.

\section{Results}

Using a zero-degree rod endoscope through a $25 \mathrm{~mm}$ retrosigmoidal craniotomy, a sufficient view of the structures to the $\mathrm{CP}$ angle was obtained. The acoustic meatus with the associated CN VII/CN VIII complex was the most prominent landmark after creating the initial craniotomy. Dissection of this region using a bimanual microsurgical technique was facile and required minimal cerebellar retraction. After establishing the CN VII/CN VIII complex as a reference point, posterior access to $\mathrm{CN}$ IX through $\mathrm{CN}$ XI was obtained without difficulty. As with the CN VII/CN VIII complex, only minimal cerebellar retraction was necessary, and a sufficiently large surgical corridor for dissection was maintained.

Moving rostrally, CN V could be accessed with little interference with the cerebellum. However, as the endoscope 
moved deeper into the $\mathrm{CP}$ angle, visualization of the caudal structures was lost. Particularly, the CN VII/VIII complex could be accidentally lesioned. In one cadaver, $\mathrm{CN}$ VIII was cut accidentally while dissecting CN IV and CN V.

Rotating the endoscope superiorly in the craniotomy and inserting it slightly inferior to the tentorium avoided interference with CN VII/VIII. However, this approach did require more manipulation of the cerebellum. Using the superior position of the endoscope was helpful for dissecting CN VI. The inferior position of CN VI placed CN VIII at greater risk for iatrogenic trauma. To gain an unobstructed view of CN VI from this vantage point required the excision of the petrosal vein, which was located posteriorly to the CN V ( - Fig. 1).

Similar views of CN V through XI were obtained via microscope (-Figs. 2-4). However, the additional retraction of the cerebellum with the a brain spatula was required. The microscopic line of sight view meant that the microsurgical instruments obscured the anatomy during dissection. This required alteration of the dissection technique and positional changes of the microscope.

Views of CN III and the pituitary stalk were unobtainable with the microscope. CN III, infundibulum, mamillary bodies,

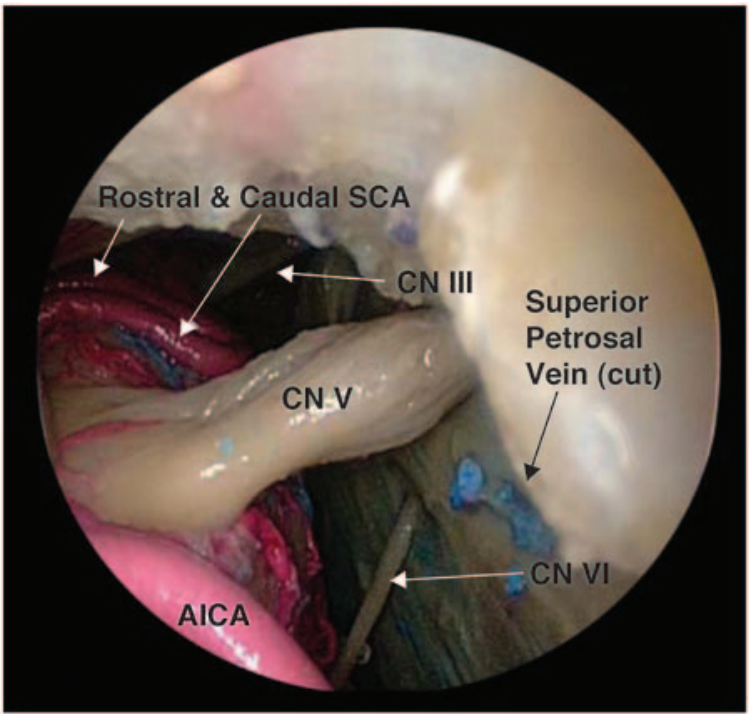

Fig. 1 Endoscopic view of the trigeminal nerve (CN V), with abducent nerve ( $\mathrm{CN} \mathrm{VI)} \mathrm{that} \mathrm{was} \mathrm{exposed} \mathrm{after} \mathrm{cutting} \mathrm{the} \mathrm{superior} \mathrm{petrosal} \mathrm{vein.}$ AICA, anterior inferior cerebellar artery; SCA, superior cerebellar artery.

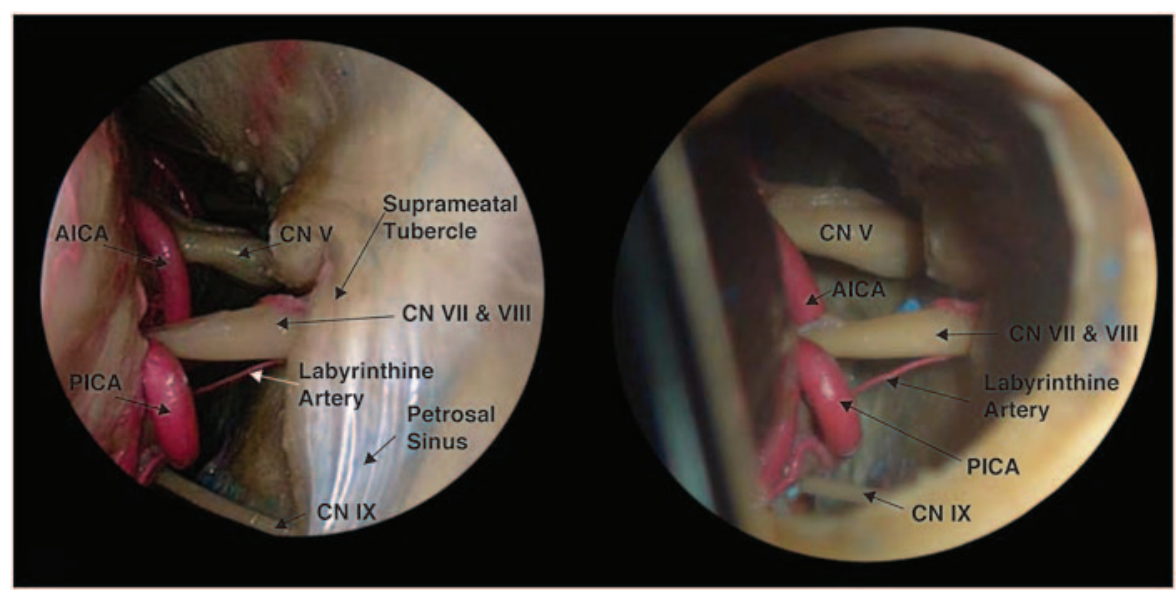

Fig. 2 Endoscopic (left) and microscopic (right) views of the trigeminal nerve (CN V) and the facial/vestibulocochlear nerve complex (CN VII and CN VIII). AICA, anterior inferior cerebellar artery; CN IX, glossopharyngeal nerve; PICA, posterior inferior cerebellar artery.

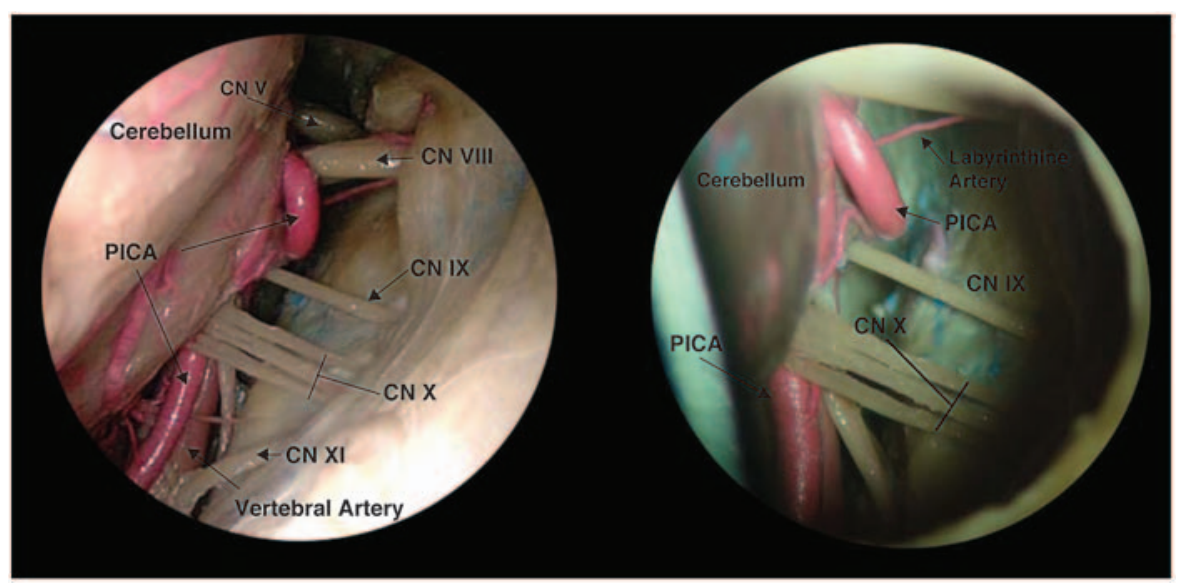

Fig. 3 Endoscopic (left) and microscopic (right) views of the lower cranial nerves. CN V, trigeminal nerve; CN VIII, vestibulocochlear nerve; CN IX, glossopharyngeal nerve; CN X, vagus nerve; CN XI, spinal accessory nerve; PICA, posterior inferior cerebellar artery. 


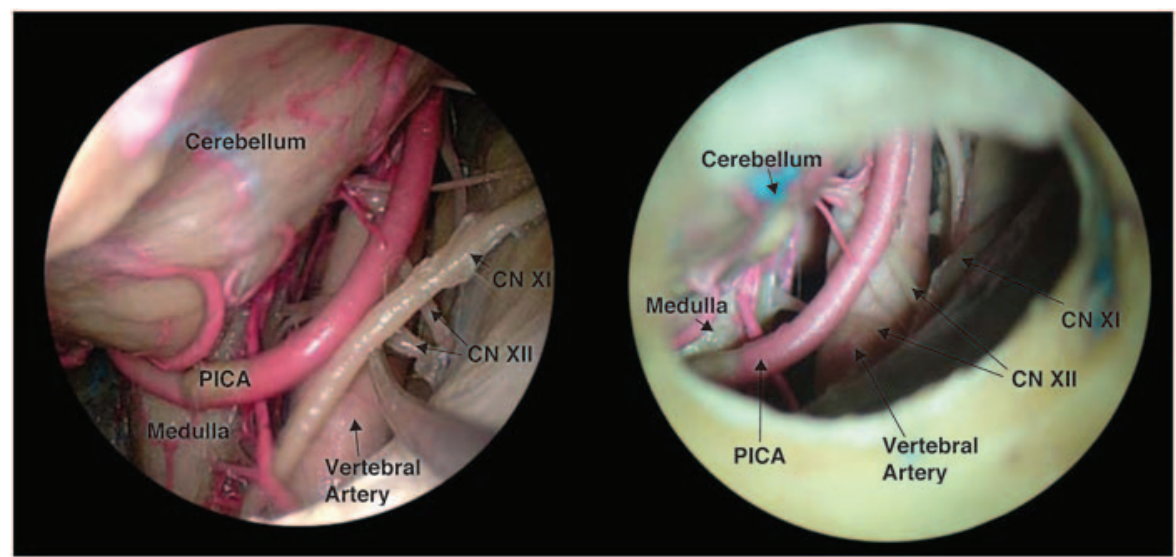

Fig. 4 Endoscopic (left) and microscopic (right) views of the spinal accessory nerve (CN XI), hypoglossal nerve (CN XII), and the posterior inferior cerebellar artery (PICA).

and clivus were all clearly visualized using the endoscope (-Figs. 5 and $\mathbf{6}$ ). Dissection of the pituitary stalk was facile; however, the working space was limited when compared with that in the $\mathrm{CP}$ angle. As with all deeper structures, caution was undertaken to prevent trauma of the caudal neurovascular structures. The surgical corridor to the clivus was larger when compared with the suprasellar region, but was still smaller than the surgical corridor to the $\mathrm{CP}$ angle.

The dissection of CN IV could be completed with both the microscope and endoscopy (-Fig. 7). However the endoscope permitted complete dissection of this nerve from the tentorium incisure to its point of origin inferior to the inferior colliculus (-Fig. 8). To permit a sufficient surgical corridor additional cerebellar retraction was required.

Views of the contralateral $\mathrm{CN} \mathrm{XI}$, and posterior medulla oblongata were obtained with the endoscope. These structures could not be visualized with the microscope (-Fig. 9). Dissections of these regions were possible, however, with the use of angled instruments. Similarly angled instruments

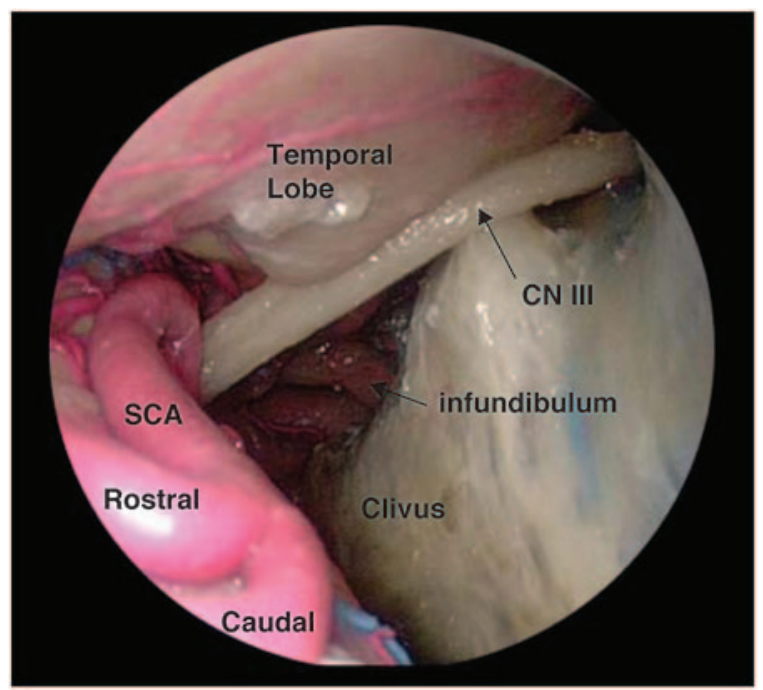

Fig. 5 Endoscopic view of the oculomotor nerve (CN III) and the rostral/caudal divisions of the superior cerebellar artery.

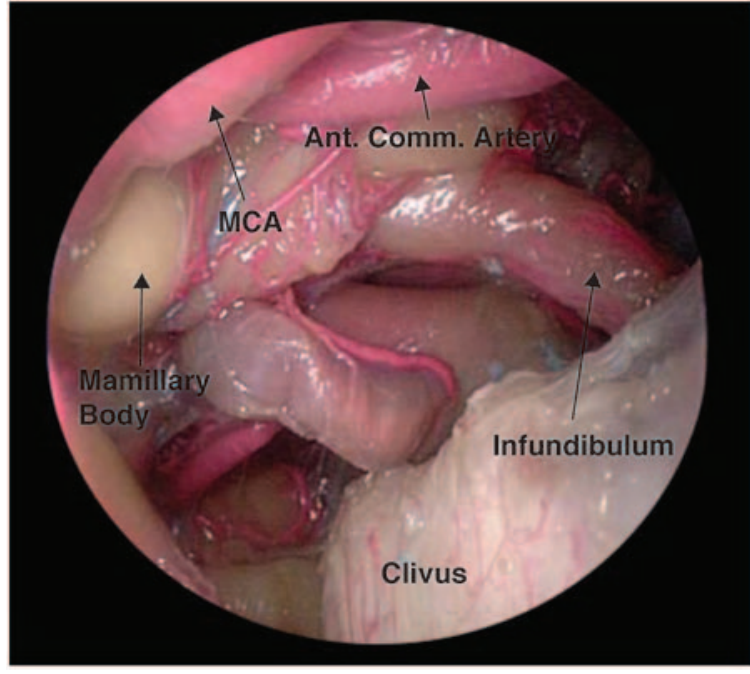

Fig. 6 Endoscopic view of the suprasellar space. Ant. Comm. Artery, anterior communicating artery; MCA, middle cerebral artery.

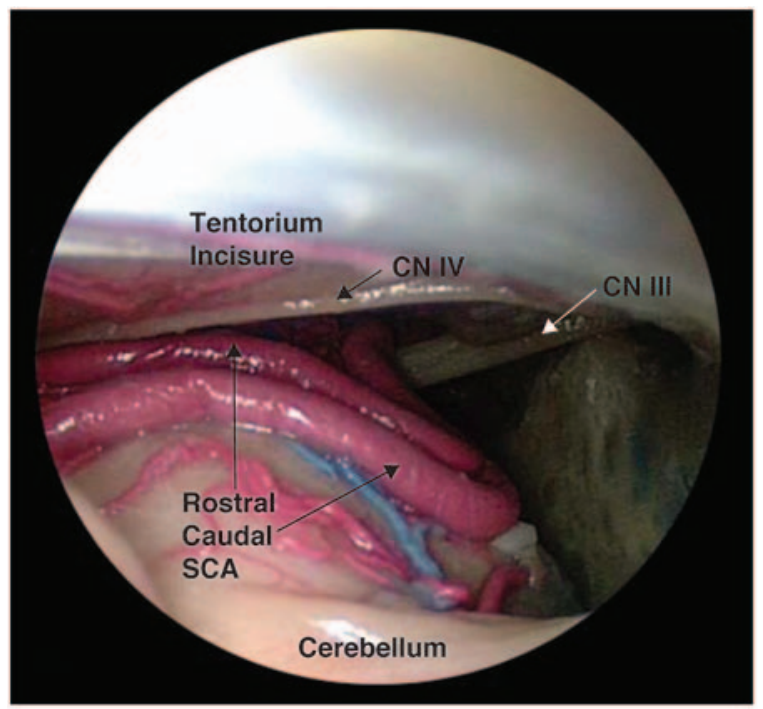

Fig. 7 Endoscopic view of the tentorium incisure with related anatomy. CN III, oculomotor nerve; CN IV, trochlear nerve; SCA, superior cerebellar artery. 


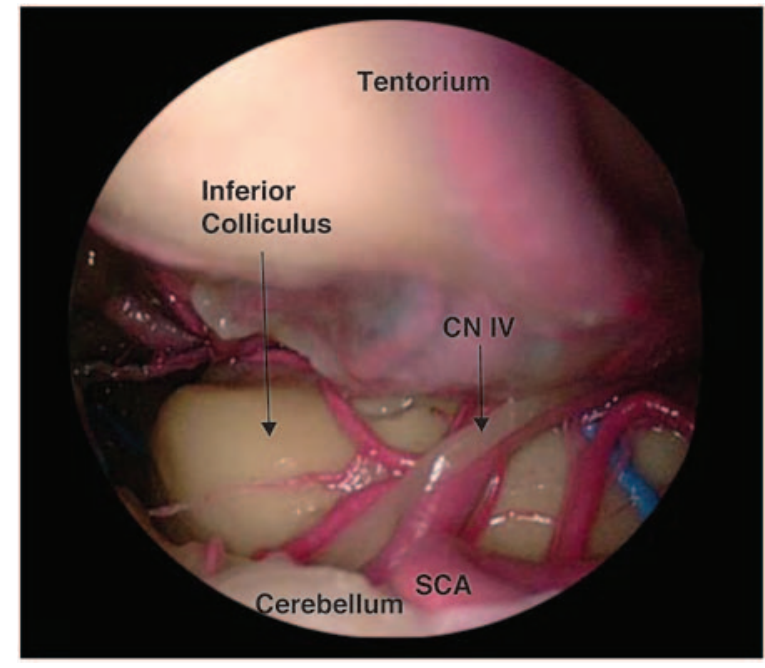

Fig. 8 Endoscopic view of the posterior midbrain. CN IV, trochlear nerve; SCA, superior cerebellar artery.

could also permit access to CN XII (-Fig. 10). However, manipulation and possible transection of CN XI could possibly be required. The roots of $\mathrm{C} 1$ were also identified with the endoscope. However, like the access to CN XII, transection of neurovascular structures would be required and thereby would limit access to this region using a retrosigmoidal entry point.

\section{Discussion}

The $\mathrm{CP}$ angle is divided into a superior and inferior limb with the lateral borders being the petrosal surface of the cerebellum as they fold around the pons and the middle cerebral peduncle. ${ }^{1,2}$ The cerebral peduncle and pons along with CN IV and $\mathrm{V}$ form the superior limb. The medulla oblongata along with CN IX, X, and the spinal accessory nerves create the inferior limb. CN VI is located at the junction of the superior

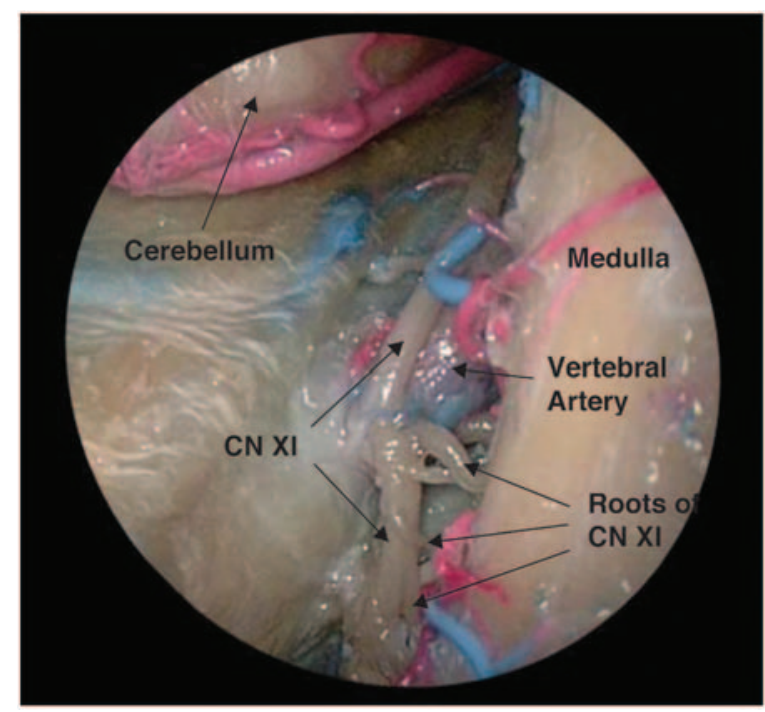

Fig. 9 Endoscopic view of the contralateral brain stem using the same retrosigmoidal craniotomy as the other figures. CN XI, spinal accessory nerve.

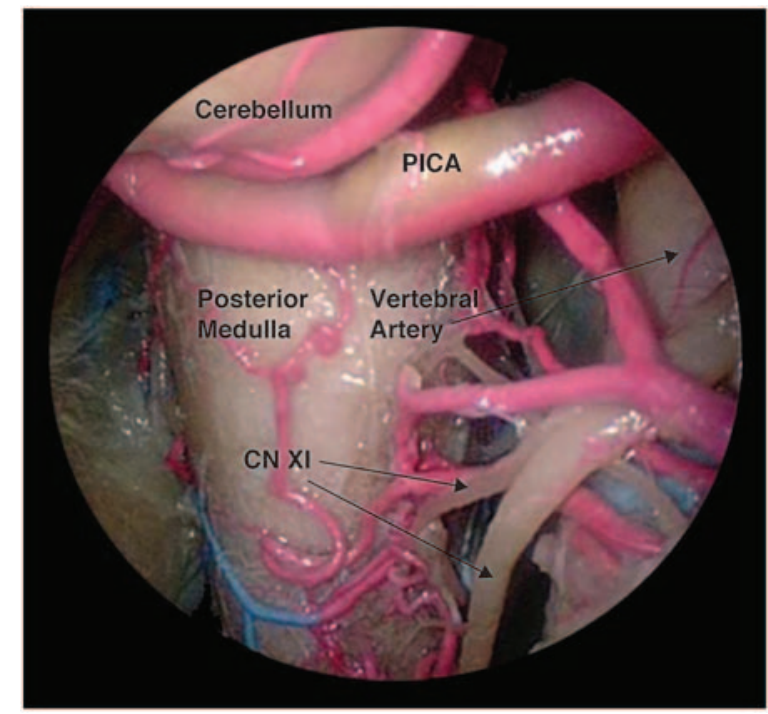

Fig. 10 Endoscopic view of the posterior medulla. CN XI, spinal accessory nerve; PICA, posterior inferior cerebellar artery.

and inferior limbs. ${ }^{2} \mathrm{CN}$ VII and VIII are also native to this junction.

Surgical access to the $\mathrm{CP}$ angle is of particular importance because of the pathology that can be found in this region. ${ }^{1-3}$ Only $10 \%$ of intradural tumors can be found in the CP angle. ${ }^{11}$ Even though the occurrence is rare, they may still necessitate surgical intervention. ${ }^{2}$ Sometimes the tumor may be fatal, which underscores the urgency for intradural surgery. ${ }^{12}$ The most common tumors of the $\mathrm{CP}$ angle are vestibular schwannomas, which account for $78 \%{ }^{13}$ Many other tumors affect this area and include meningiomas, epidermoid tumors, arachnoid cysts, and lipomas. 1,2,14,15

Neurovascular compression syndromes may also necessitate surgical access to the $\mathrm{CP}$ angle. ${ }^{2,3,5,16}$ Arterial compression of the trigeminal nerve is a cause of trigeminal neuralgia and can be relieved via surgical decompression. Similarly, surgical decompression techniques may be applied to abducens and the vestibulocochlear neurovascular complexes. ${ }^{2,3}$ The retrosigmoidal approach also permits lateral access to the anterior inferior cerebral artery, which may also create compressive symptoms of the facial nerve. ${ }^{2,17}$

The traditional retrosigmoidal approach to the CP angle uses a microscope for visualization. This has necessitated a larger surgical window to allow an unobstructed view of the targeted neurovascular structures. ${ }^{4}$ The craniotomy needs to be sufficiently large to view deep into the midline of the posterior fossa. ${ }^{4}$ To achieve the adequate visualization, retraction of the cerebellum is necessary. ${ }^{4}$ Minimization of brain manipulation and retraction is desirable because compression causes decreased cerebral perfusion. ${ }^{5-7}$ This increases the risk of infarction and other morbidities. ${ }^{6,7}$

The development of the endoscope has permitted the visualization of deep-seated structures without the need for highly invasive open surgeries. Endoscopic technology is now widely used by many surgical disciplines. Otolaryngology and neurosurgery have successfully applied the technology to create a variety of innovative skull base approaches. ${ }^{8}$ These 
endoscopic approaches have helped improve patient outcomes and are, in some cases, an attractive alternative to open surgery.

The endoscope allows to visualize the targeted anatomy directly, allowing to use minimized surgical corridors. This contrasts with microscopic approaches where structures obstructing the view on the targeted lesion needs to be reflected or excised. Endoscopy has the ability to obviate the morbidity associated with the manipulation and removal of vital tissue. It is for this reason that the endoscope has assisted the surgeon to obtaining direct visualization of structures and pathologies of the skull base. These advantages have provided the impetus for the increasing use of the endoscope.

When approaching the CP angle, it is important to avoid sinus bleeding through correct placement of the initial craniotomy. A properly placed craniotomy also permits the minimization of cerebellar retraction and a direct path to the $\mathrm{CP}$ angle. Placing the craniotomy posterior to the sigmoid sinus and inferior to the transverse sinus prevents hemorrhaging complications. Raso and Gusmao provided guidelines for the optimal placement of a retrosigmoidal craniotomy. ${ }^{10}$ Using these guidelines, the location of the intersection of the sinuses appeared to be consistently the same when examining multiple dry skulls.

In this study, using a zero-degree rod endoscope through a retrosigmoidal craniotomy, a sufficient view of the structures to the $\mathrm{CP}$ angle was obtained. Dissection of the ipsilateral side gave clear visualization of cranial nerves III to XII with the associated vasculature. Additionally, a view of CN IV attaching to the midbrain inferior to the inferior colliculus was possible. The exposure could be further expanded into the middle fossa by drilling the suprameatal extension located posterior to $\mathrm{CN} \mathrm{V}$ and thereby permitting access to posterior section of Meckel's cave. ${ }^{2,17}$ Directing the endoscope anterior to the $\mathrm{CP}$ angle along the clivus allowed visualization of the infundibulum extending inferiorly into the sellae. The surgical corridor to the clival region can also be expanded further when combined with the endonasal approach. In addition, views of the contralateral CN XI and posterior medulla oblongata were obtained, although dissection in this area was challenging. The visualization obtained with the endoscope was superior to that obtained with the microscope. Thus, from an anatomical perspective, it is feasible to access deep structures in the posterior fossa using a keyhole craniotomy and endoscopic visualization. It is important to understand the limitations of the technology. One limitation is lacking visualization of structures behind the tip of the endoscope. Awareness of this limitation and a thorough knowledge of the anatomy will prevent injury to important neurovascular structures that are not in direct view during surgery.

\section{Conclusion}

A retrosigmoidal endoscopic approach to the midline structures in the posterior fossa is anatomically feasible. The morbidity associated with retraction of the cerebellum could possibly be avoided, potentially leading to better patient outcomes. Retrosigmoidal endoscopy provides access to anatomical structures which would not have been possible using a microscope in a suboccipital approach. Further understanding of the endoscopic anatomy of the posterior fossa can allow for advances in cranial base surgery with improved safety and efficacy.

\section{Conflict of Interest \\ None}

\section{References}

1 Brackmann D, Crawford J, Green JD Jr. Cerebellopontine angle tumors. In: Bailey B, Johnson J, Newlands S, eds. Head \& Neck Surgery-Otolaryngology. 4th ed. New York: Lippincott Williams \& Wilkins; 2006:2208-2227

2 Rhoton AL Jr. The cerebellopontine angle and posterior fossa cranial nerves by the retrosigmoid approach. Neurosurgery 2000;47(3, Suppl):S93-S129

3 Shahinian H. Skull base surgery. In: Thorne C, Beasley R, Aston S, Bartlett S, Gurtner G, Spear S, eds. Grabb and Smith's Plastic Surgery. 6th ed. New York: Lippincott Williams \& Wilkins; 2007:348-349

4 Shahinian H, Kabil M, Jarrahy R, Thill M. Endoscopic Skull Base Surgery: A Comprehensive Guide with Illustrative Cases. Totowa, NJ: Humana Press; 2008:109-171

5 Fries G, Perneczky A. Endoscope-assisted brain surgery: part 2analysis of 380 procedures. Neurosurgery 1998;42(2):226-231, discussion 231-232

6 Yokoh A, Sugita K, Kobayashi S. Intermittent versus continuous brain retraction. An experimental study. J Neurosurg 1983;58(6): 918-923

7 Arbit E, DiResta GR, Bedford RF, Shah NK, Galicich JH. Intraoperative measurement of cerebral and tumor blood flow with laser-Doppler flowmetry. Neurosurgery 1989;24(2):166-170, 198

8 Kassam A, Snyderman CH, Mintz A, Gardner P, Carrau RL. Expanded endonasal approach: the rostrocaudal axis. Part I. Crista galli to the sella turcica. Neurosurg Focus 2005;19(1):E3

9 Sanan A, Abdel Aziz KM, Janjua RM, van Loveren HR, Keller JT. Colored silicone injection for use in neurosurgical dissections: anatomic technical note. Neurosurgery 1999;45(5):1267-1271, discussion 1271-1274

10 Raso J, Gusmao S. A new landmark for finding the sigmoid sinus in suboccipital craniotomies. Neurosurgery 2011;68(1 suppl operative):1-6

11 Comey CH, McLaughlin MR, Jho HD, Martinez AJ, Lunsford LD. Death from a malignant cerebellopontine angle triton tumor despite stereotactic radiosurgery. Case report. J Neurosurg 1998;89(4):653-658

12 Gerganov VM, Romansky KV, Bussarsky VA, Noutchev LT, Iliev IN. Endoscope-assisted microsurgery of large vestibular schwannomas. Minim Invasive Neurosurg 2005;48(1):39-43

13 Schroeder HW, Oertel J, Gaab MR. Endoscope-assisted microsurgical resection of epidermoid tumors of the cerebellopontine angle. J Neurosurg 2004;101(2):227-232

14 Rak R, Sekhar LN, Stimac D, Hechl P. Endoscope-assisted microsurgery for microvascular compression syndromes. Neurosurgery 2004;54(4):876-881, discussion 881-883

15 Mangham CA Jr. Retrosigmoid versus middle fossa surgery for small vestibular schwannomas. Laryngoscope 2004;114(8):1455-1461

16 Catapano D, Sloffer CA, Frank G, Pasquini E, D’Angelo VA, Lanzino G. Comparison between the microscope and endoscope in the direct endonasal extended transsphenoidal approach: anatomical study. J Neurosurg 2006;104(3):419-425

17 Ong YK, Solares CA, Carrau RL, Snyderman CH. New developments in transnasal endoscopic surgery for malignancies of the sinonasal tract and adjacent skull base. Curr Opin Otolaryngol Head Neck Surg 2010;18(2):107-113 